\title{
ROTATION-SHEARING INTERFEROMETRY
}

François Roddier

Département d'Astrophysique de l'I.M.S.P., Equipe de recherche associée au C.N.R.S., Université de Nice, France.

\section{ABSTRACT}

Holograms of incoherent objects can be recorded with a rotationshearing interferometer allowing corrections of wavefront errors. Holographic images taken in our laboratory, either through aberrated optics or atmospheric turbulence, have been successfully restored. A stellar hologram is presented.

\section{INTRODUCTION}

In optical astronomy, images are highly degraded by atmospheric turbulence. The diameter of the seeing disk may vary from 0.2 arcseconds to 10 arcseconds or more according to weather conditions. A typical diameter is of the order of 2 arcseconds which is the angular resolution of a $6 \mathrm{~cm}$ lens at the diffraction limit.

Large: telescopes have been built for their light collecting capabilities, not for their resolving power which is, in general, of the order of 0.2 arcseconds, that is far from the diffraction limit.

Up to now, there have been several approaches to overcome this situation. Three kinds of approach may be distinguished :

a) Postdetection processing of the image : Usual deconvolution procedures have been unsuccessful for two reasons : the exponential decrease of the modulation transfer function for long exposures and the random properties of the short exposures. A considerable improvement has been brought by Labeyrie ${ }^{1}$ who applied the speckle-interferometric technique to the statistical analysis of short exposure sequences. However such a procedure gives only the autocorrelation or power spectrum of the image. Obtaining an image is not straightforward in the general case. 
b) Imaging through pupil masks : The double aperture of the Michelson stellar interferometer ${ }^{2}$ was the first mask to be used. Russel and Goodman ${ }^{3}$ have investigated the use of more elaborated non redundant masks. The main disadvantages of these methods are that the image Fourier components are measured sequentially and most of the light is lost.

c) Active optics : This is certainly the most promising technique at high light levels. However Goodman ${ }^{4}$ has shown that at low 1 ight levels such as those encountered in Astronomy, photon noise introduces errors which make passive methods superior to active ones

\section{IMAGING WITH A COHERENCE INTERFEROMETER}

Our approach is of a fourth kind. It consists in postdetection processing of interferograms in the pupil space. It is somewhat similar to Michelson interferometry but allows us to observe all the Fourier components of the image at the same time. It is equivalent to a great number of Michelson interferometer working in parallel. This technique is also called incoherent holography ${ }^{5,6}$. It consists in observing the interference pattern produced by two superimposed images of the telescope pupil, one being rotated with respect to the other. In order to do this, we use a rotation shearing interferometer ${ }^{7}$ also called coherence interferometer ${ }^{8}$. It is schematically represented on fig. 1 .

The mechanism of this interferometer is best explained when prism $A$ is rotated by an angle of ninety degrees which corresponds to a one hundred and eighty degrees rotation of the image. In this case, the interferometer gives two images of the object symetrically displayed around the axis. Each point source in the object gives rise to fringes of equal thickness with different period and orientation. Since the object is incoherent, all these fringe systems will add in intensity producing a Fourier transform of the intensity distribution in the object

The maximum resolution corresponding to the pupil frequency cut off is obtained in this case. For a smaller rotation angle, a magnification of the hologram is observed and the cut off frequency is reduced ${ }^{9}$. By demagni- 


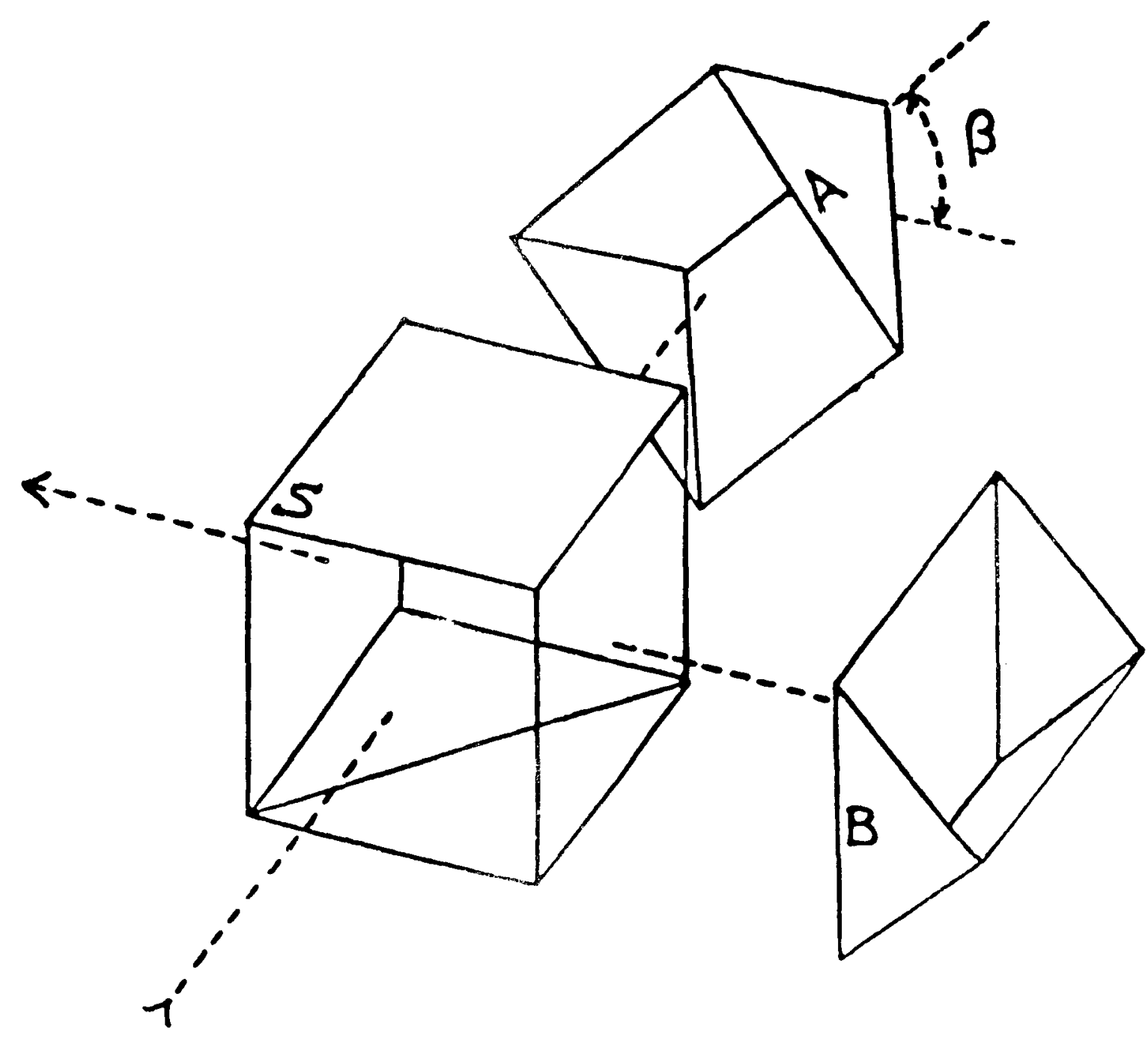

Figure 1. Perspective view of a rotation shearing interferometer A: Rotatable roof-prism; B: Fixed roof-prism;

S: Beam splitter. 
fying the image a higher luminosity is obtained at the expense of the resolving power so that the best compromise between luminosity and resolution can always be achieved.

In order to get maximum contrast fringes for any rotation angle, the polarisation states of the two interfering beams must be matched. This has been successfully achieved by cementing appropriate phase plates on the entrance face of our roof prisms 10

Under usual conditions, the bandwidth must not exceed $50 \AA$. We have been able to use a bandwidth 10 times larger by putting a chromatic optical system which gives a magnification proportional to the wavelength before the interferometer

Incoherent holograms behave like usual holograms since a restitution of the image can be obtained by observing their Fraunhöfer diffraction pattern. Fig. 2 shows an example of such a restitution made in our laboratory by F.Martin. In fact, in this case, the squared modulus of the object intensity is restored.

One of the main problems encountered with incoherent holography is the decrease of the signal to noise ratio as the square root of the number of pixels in the image ${ }^{11}$. However, when dealing with highly degraded images we shall see that the signal to noise ratio can be higher in the Fourier plane than in the image plane.

\section{IMAGING THROUGH FIXED ABERRATIONS}

We shall first examine the problem of restoring images degraded by fixed aberrations due to bad optics. This is done by using a reference point source within the isoplanatic patch.

When done in the image plane, the procedure is known as deconvolution. The optical transfer function is obtained by computing the Fourier transform of the recorded point spread function. In the case of strong aberrations, this transfer function is highly attenuated everywhere except near the origin and goes down to zero at several frequencies where the information is definitely lost (Fig. 3). The average attenuation factor is known to be of the 


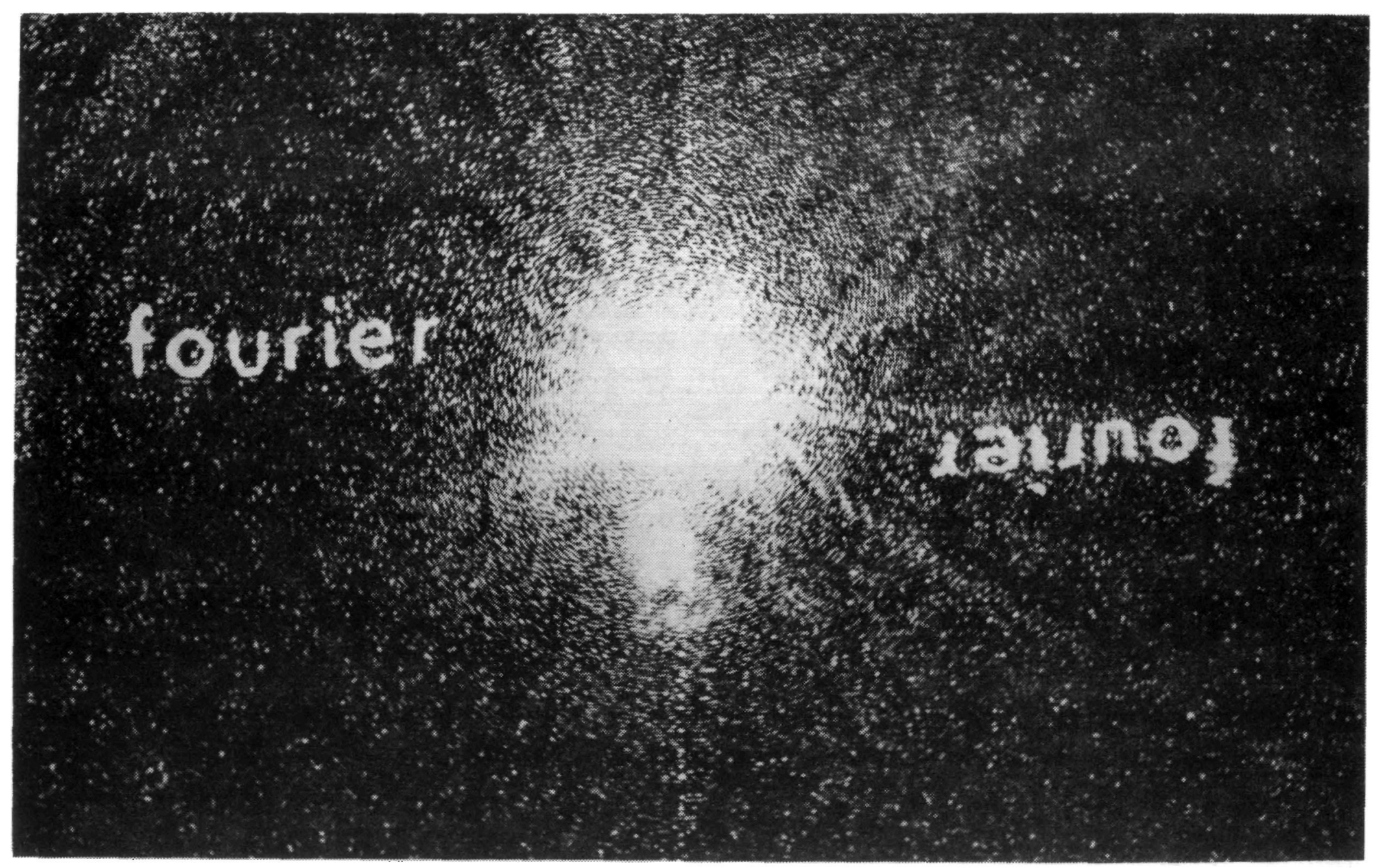

Figure 2. Diffraction pattern of an incoherent hologram of the word "Fourier".

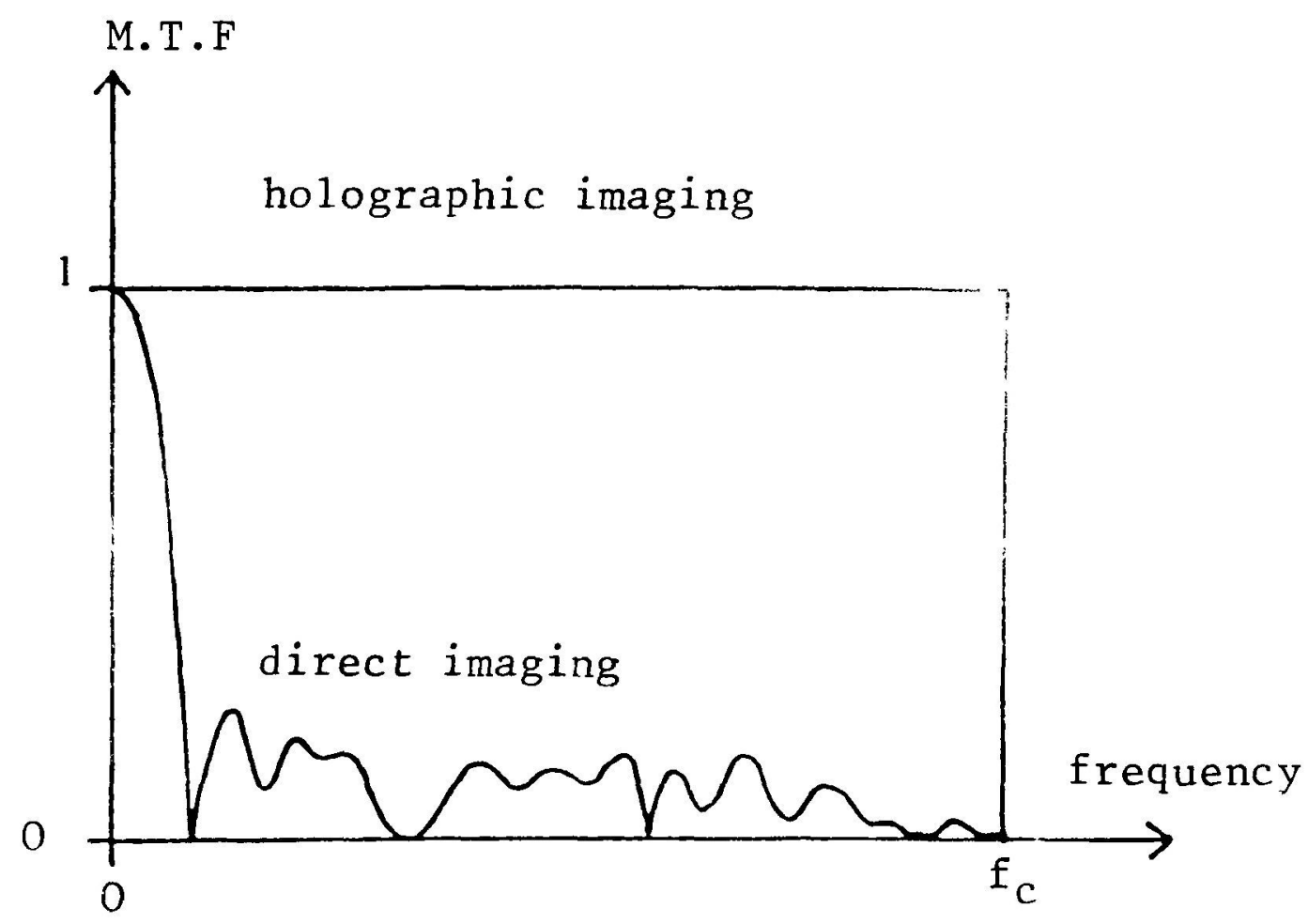

Figure 3. Modulation transfer function (M.T.F.) for direct and holographic imaging through aberrated optics. 
order of $\sqrt{N}$, where $N$ is the number of speckles in the point spread function ${ }^{12}$. The signal to noise ratio is affected by the same factor. The net result is that deconvolution is difficult and the image can only be partially restored.

On the other hand, incoherent holography allows direct recording of the complex optical transfer function without any attenuation at any frequency (Fig. 3). Indeed the fringe amplitude is not affected by optical aberrations as we shall see in a simple example.

Fig. 4 shows a hologram of a simulated double star (two point sources). The two associated fringe systems give rise to a Moiré pattern. The beat frequency and direction give the space between the stars and their orientation.

Fig. 5 shows a hologram of the same source taken through strongly aberrated optics. The fringes are highly distorted. Under these conditions the double star was not resolved. However, since the two fringe patterns are distorted in the same way, the resulting Moiré pattern remains unaffected.

Such information is lost in the image space as shown in fig. $6^{*}$ obtained by computing the 2-dimensional Fourier transform of the intensity recorded in the hologram presented in fig. 5. However, the amount of fringe distorsion can be calibrated by recording an hologram of a single star (reference point source) under the same conditions. After subtraction of the phase errors a good image is obtained (Fig. 7). Computations were made at the "Centre de Dépouillement des clichés Astronomiques" in Nice by C.Roddier Evidently, such a perfect restoration could not have been done from photographs taken in the image plane.

We have seen that the signal to noise ratio in our incoherent holograms decreases as the square root of the number $M$ of resolved pixels in the image. With more complicated objects the restoration would not have been as good. Comparison with the average signal to noise ratio in image plane recording shows that incoherent holography remains better than usual deconvolution as

* Figures 6 to 13 inclusive are grouped together at the end of the paper on pages $32-11$ to $32-15$ inclusive. 


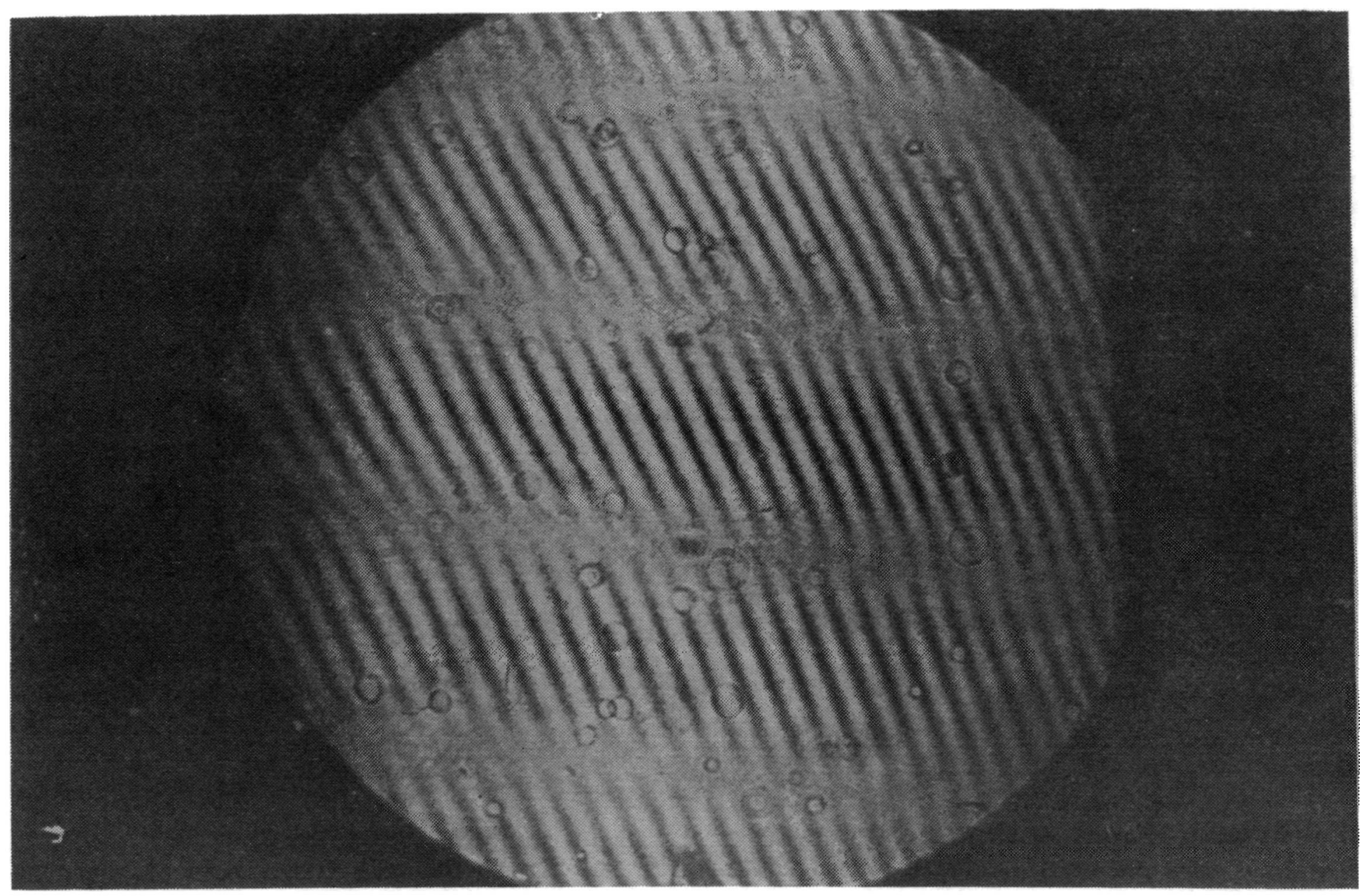

Figure 4. Incoherent hologram of a double point source through good optics.

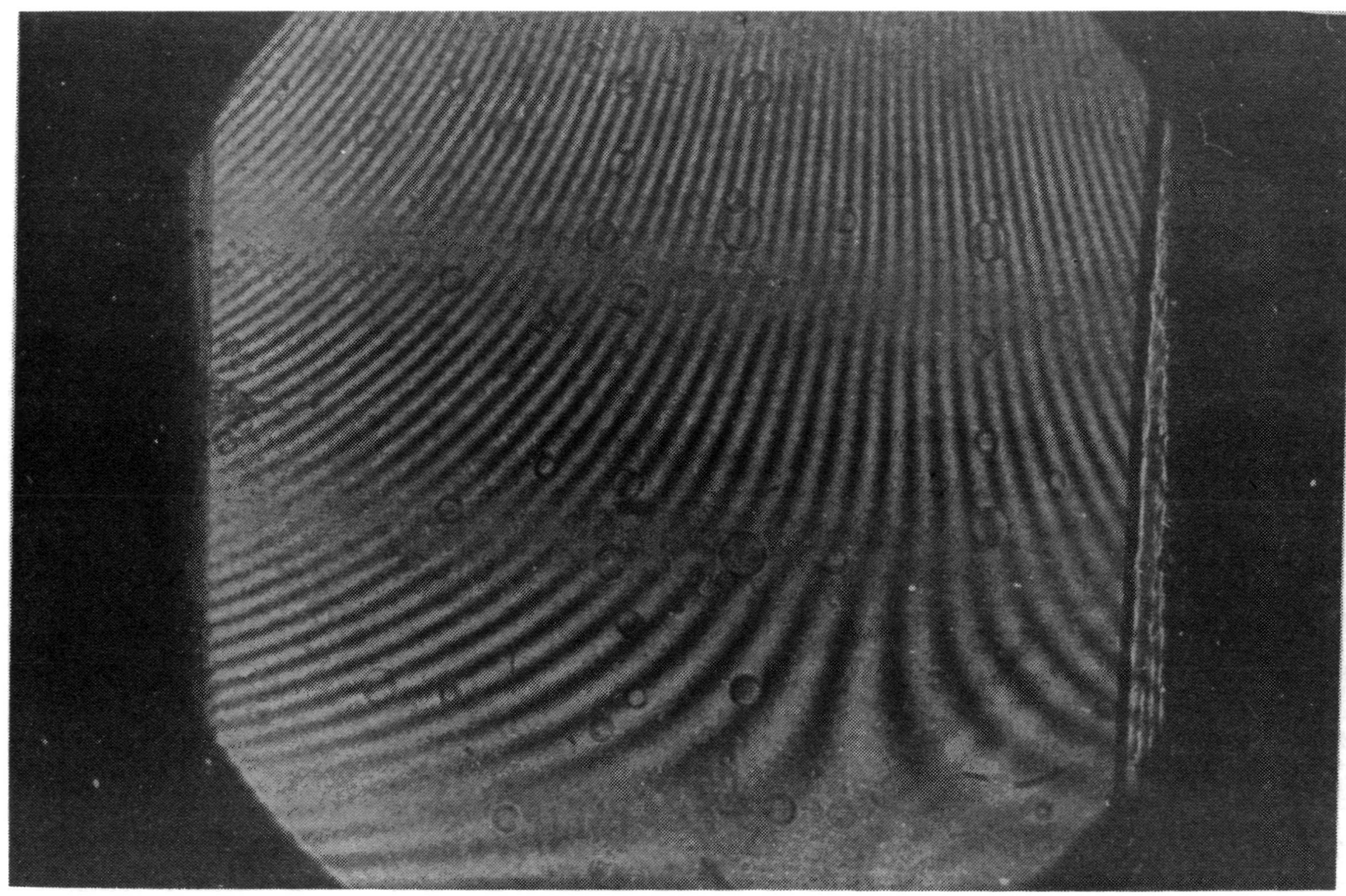

Figure 5. Incoherent hologram of a double point source through strongly aberrated optics. 
long as the number of resolved pixels is smaller than the number of specklies in the point spread function, that is

$$
M<N
$$

This is obviously the case for a strongly aberrated image of a double point source.

\section{IMAGING THROUGH TURBULENCE}

Let us now examine the case of random aberrations such as those produced by atmospheric turbulence.

By standard speckle interferometry techniques, one obtains only the amplitude of the Fourier transform of the object. However McGlamery ${ }^{13}$ and more recently Knox and Thompson ${ }^{14}$ in the United States, have shown that the phase can also be estimated by computing the average phase over all the Fourier transforms of each image. However, one must take care because the phase excursions due to turbulence increase with spatial frequency and may become much larger that $2 \pi$. Therefore the phase must be followed with continuity from zero spatial frequency.

The same procedure can be used with a sequence of turbulence distorted holograms. It consists in estimating the average fringe position in order to produce a restored hologram, the Fourier transform of which gives the restored image.

We have first taken holograms of a point source in our laboratory through artificial turbulence generated by a toaster In order to freeze the fringe distortion due to turbulence, the exposure time was of the order of a millisecond.

Fig. 8 shows the holographic image of a point source taken without turbulence. Fig. 9 shows a typical holographic image obtained through turbulence. From a sequence of nine distorted holograms, C.Roddier has computed a restored hologram. Fig. 10 shows the corresponding restored image which is almost as good as the original. 
We did the same thing with a slightly more complicated object : a double point source. Fig. 11 shows a typical holographic image from a sequence of 5 holograms. None of the images shows a resolved structure. However, the double point source is clearly resolved on the restored image with a correct ratio of intensities between the two point sources (Fig. 12).

\section{ASTRONOMICAL APPLICATIONS}

The experiment has been performed in July on real stars with the $1.52 \mathrm{~m}$ telescope of the Haute Provence Observatory in cooperation with C.Roddier and F.Martin. Fig. 13 shows a typical hologram of an unresolved star. Fringe distortion, due to turbulence, is frozen by a lms exposure. The two pupil images are moved aside in order to record the central part of the hologram otherwise hidden by the telescope central obstruction. The pupil images are blurred by the chromatic optical system.

Imaging disks of bright stars should be possible with a larger telescope. The same technique should also apply to the observation of fine structures of the solar photosphere.

\section{REFERENCES}

1) A. Labeyrie, Astron. Astrophys., 6, 85, 1970.

2) A.A. Michelson, Astrophys. J, 51, 257, 1920

3) F.D. Russe1 and J.W. Goodman, J. Opt. Soc. Am., 61, 182, 1971.

4) J.W. Goodman and J.F. Belsher, Joint Automatic Control Conference San Francisco (June 22-24), 1977.

5) G.W. Stroke and R.C. Restrick, Applied Physics Letters, 7, 9, 1965.

6) G. Cochran, J Opt. Soc. Am., 56, 1513, 1966.

7) J.D. Armitage, Jr and A. Lohmann, Optica acta, 12, 185, 1965.

8) J.B. Breckinridge, Applied Optics, 11, 2996, 1972.

9) S. Lowentha1, J. Serres et C. Froehly, C.R. Acad. Sc. Paris, 268, B 841 , 1969. 
10) F. Roddier, C. Roddier and J. Demarcq, J. of Optics (to appear in Ju1y), 1978.

11) S. Lowenthal, J. Serres and H. Arsenault, Optics Commun., 1, 438, 1970.

12) D. Korff, G. Dryden and M.G. Miller, Optics Commun., 5, 187, 1972.

13) B.L. McGlamery, N.A.S.A. Tech. Report SP-256, 167, 1971.

14) K.T. Knox and B.J. Thompson, Astrophys. J., 193, L 45, 1974. 


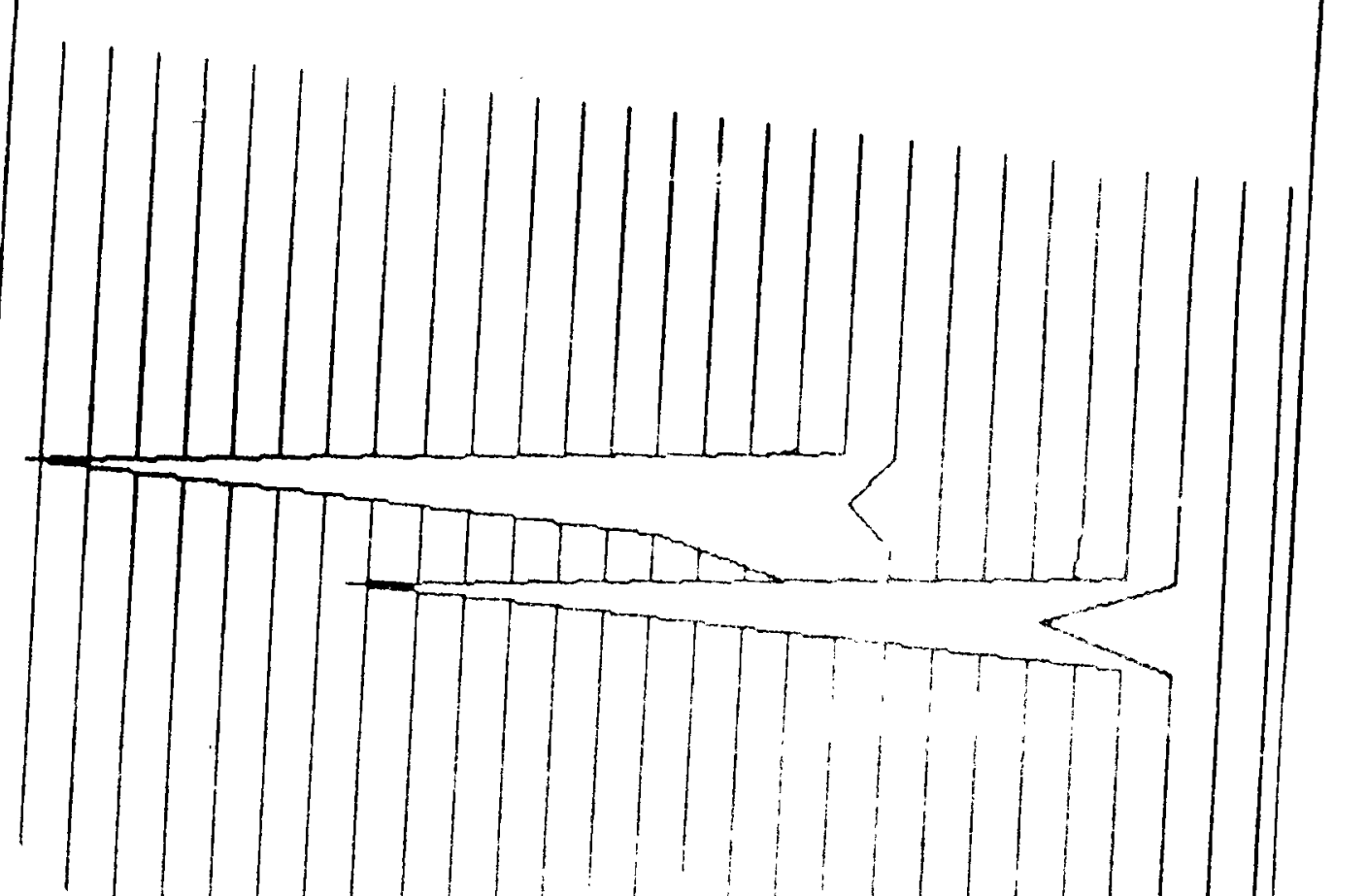

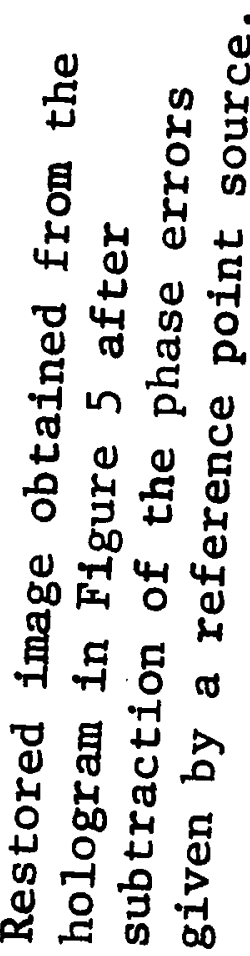

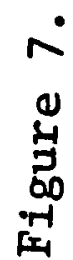

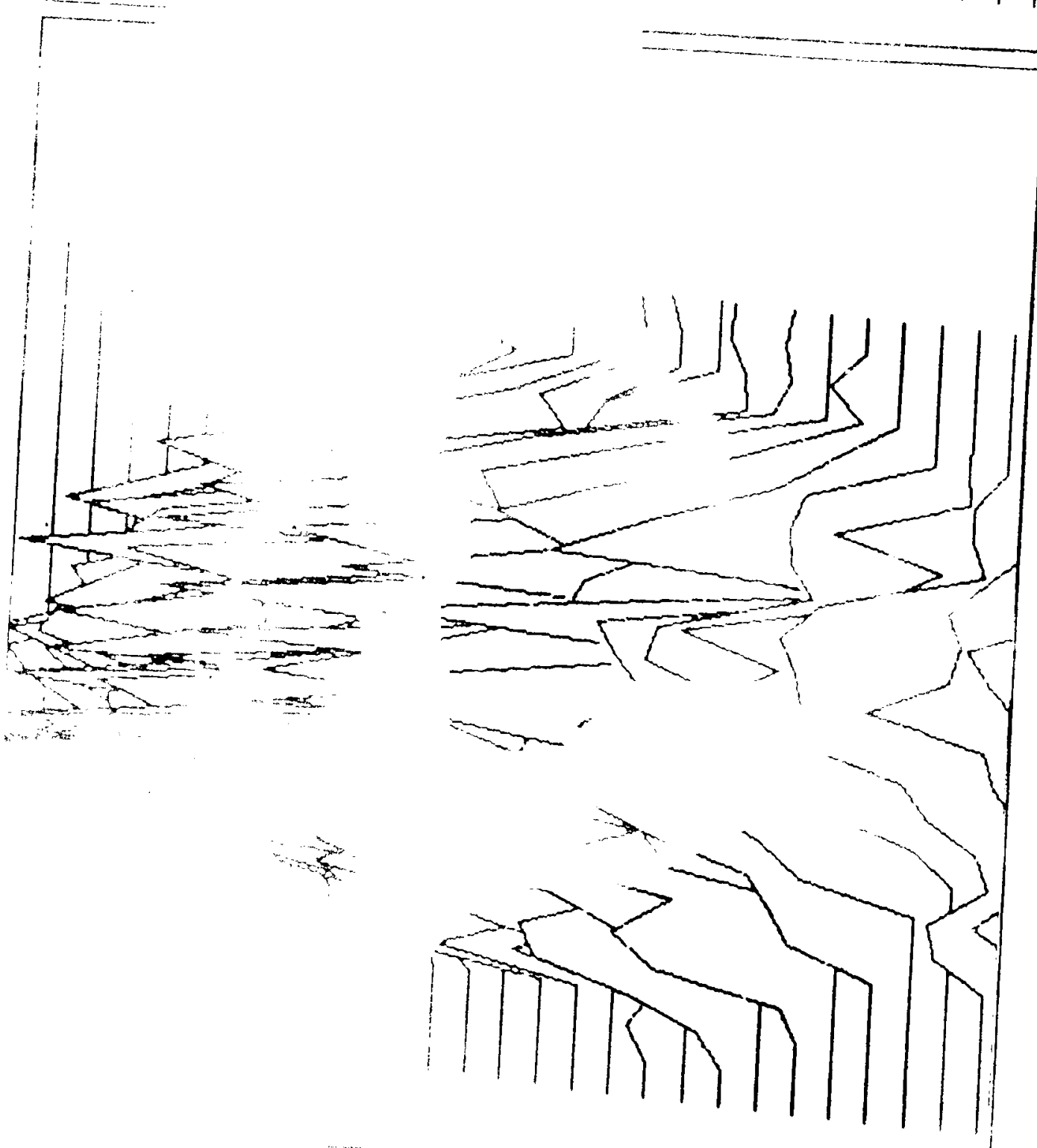

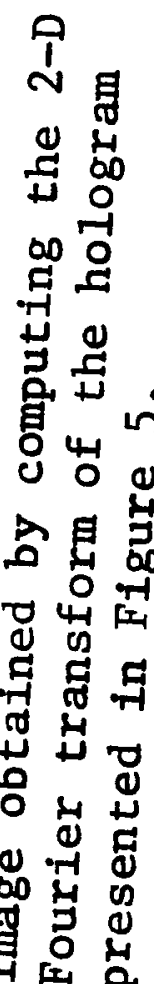

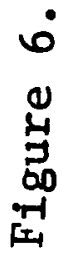



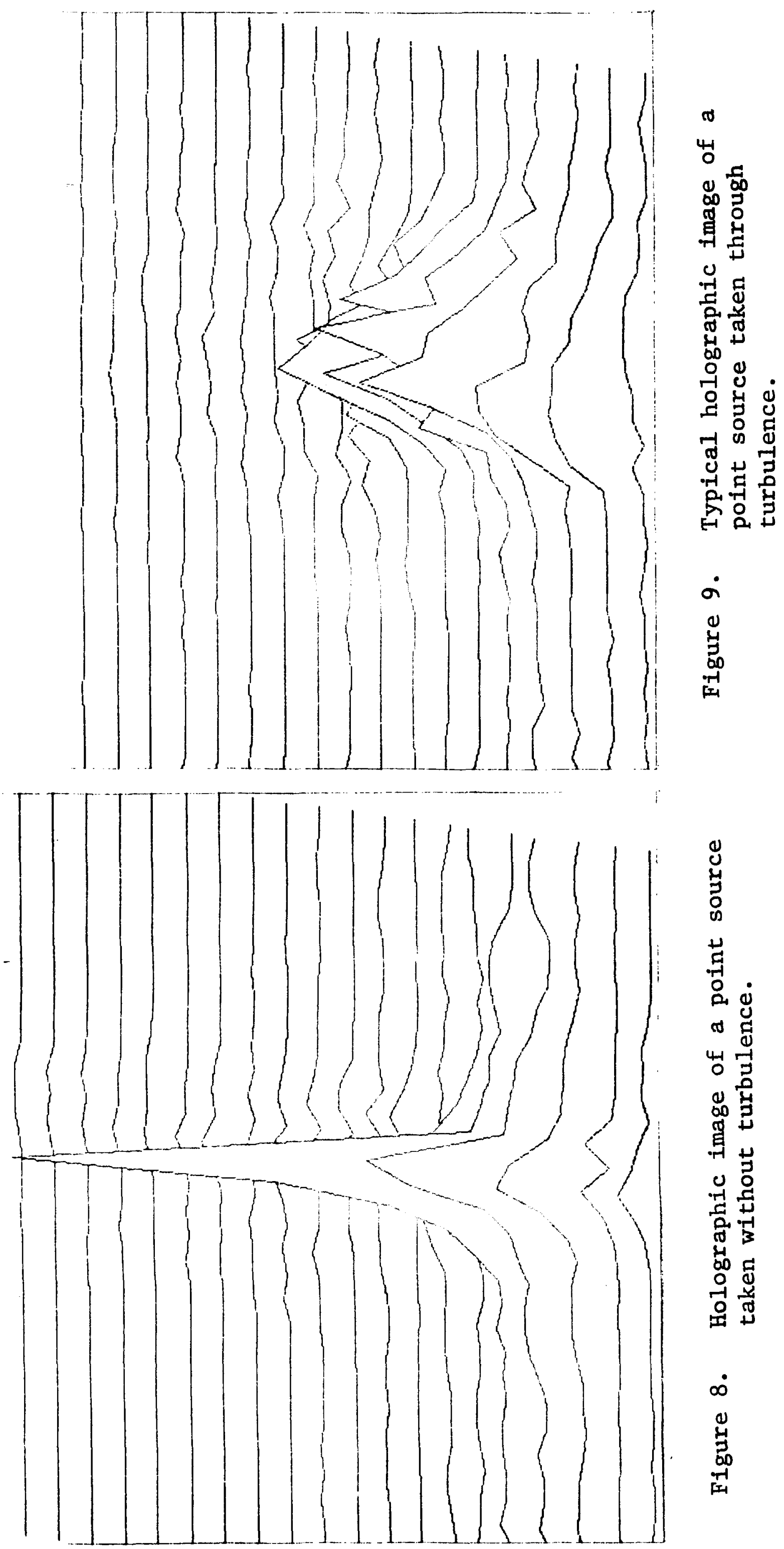


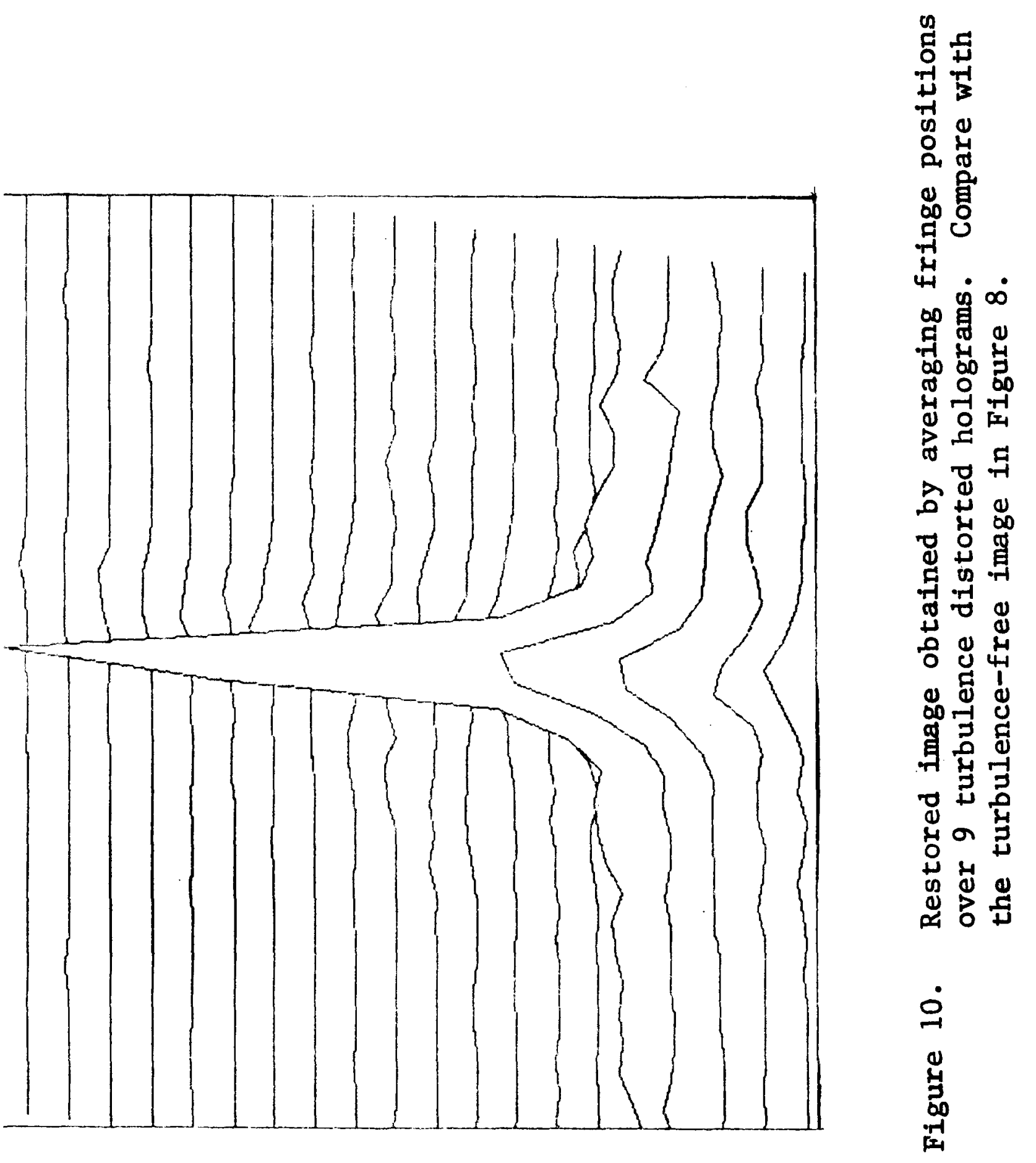



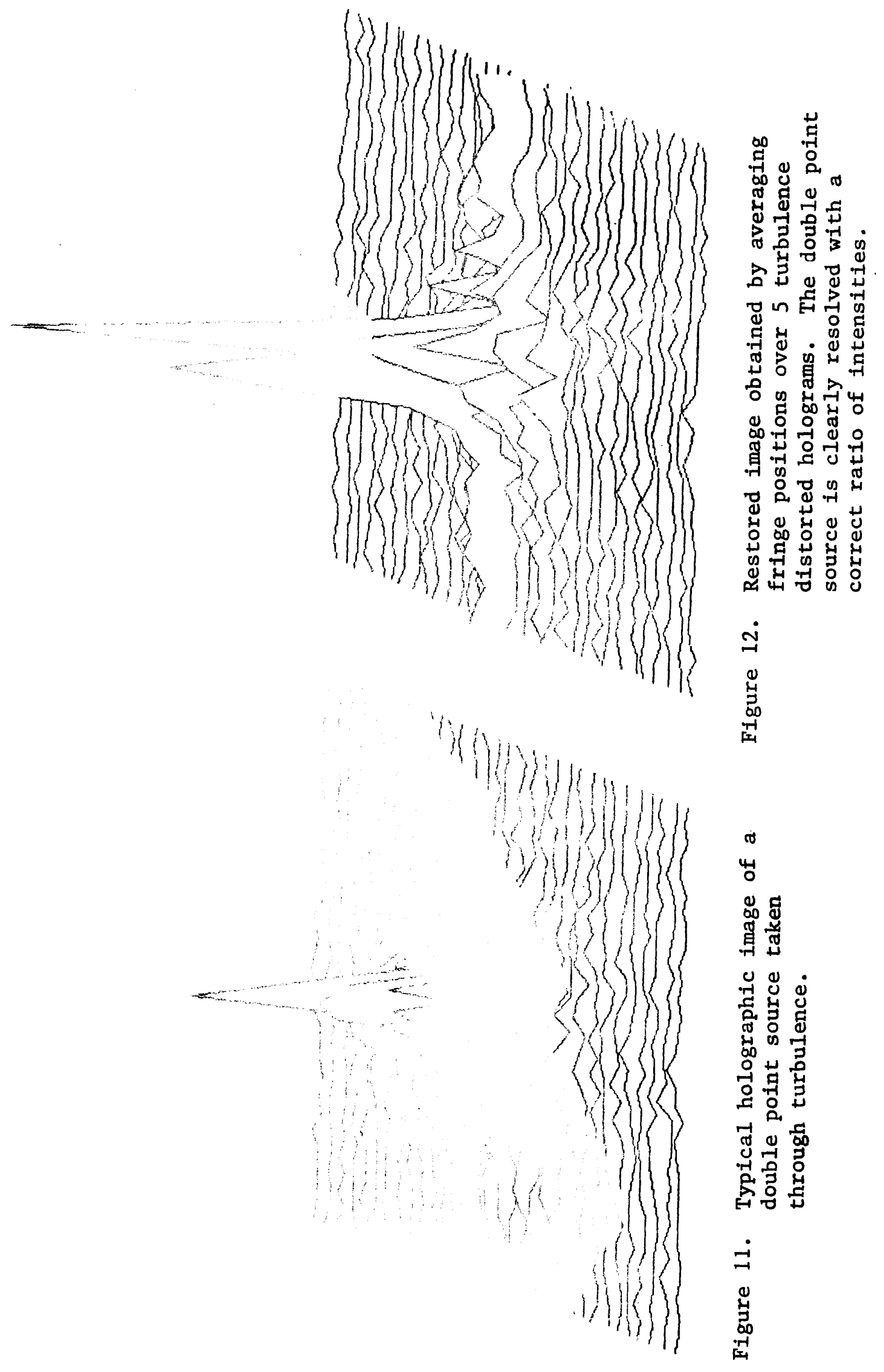


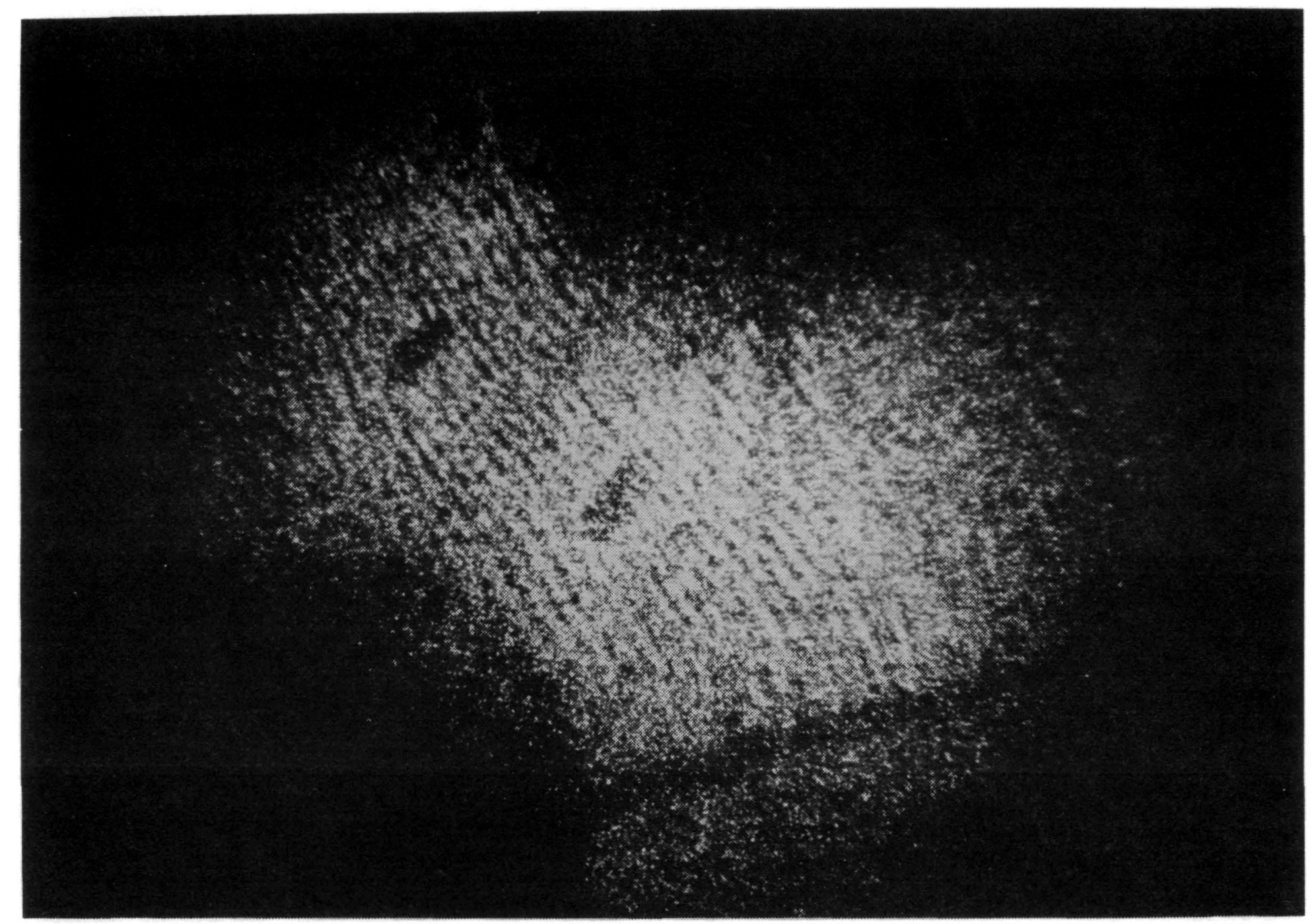

Figure 13. Example of a stellar hologram. The star is not resolved by the $1.5 \mathrm{~m}$ aperture. 